\title{
EMBARAZO ECTÓPICO OVÁRICO: REPORTE DE CASO Y REVISIÓN DE LA LITERATURA
}

\author{
Ectopic ovarian pregnancy: case report and \\ review of the literature
}

Fabio Aníbal Gómez-Rey, $M D^{1}$; Carlos Andrés Trejos-Ramírez, $M D^{2}$

Recibido: mayo 21/15 - Aceptado: octubre 2/15

\section{RESUMEN}

Objetivo: reportar un caso de embarazo ectópico ovárico y realizar una revisión de la literatura acerca del diagnóstico y su tratamiento.

Materiales y métodos: se reporta el caso de un embarazo ectópico ovárico en una paciente usuaria de dispositivo intrauterino (DIU) de cobre. Fue tratado por laparoscopia, en una institución general privada de tercer nivel de complejidad. Se realiza búsqueda de la información objeto de la revisión en las bases de datos PubMed, Ovid, ScienceDirect y Lilacs, mediante terminología MeSH: "pregnancy, ectopic"; "pregnancy, ovarian"; "pregnancy complications". Se buscaron reportes y series de caso y artículos de revisión.

Resultados: se encontraron 23 referencias bibliográficas de las cuales 1 es un capítulo de libro, 9 son revisiones de tema y 13 son reportes o series de casos. Para el diagnóstico se requiere un alto grado de sospecha clínica, medición de la subunidad beta de la hormona gonadotropina coriónica humana ( $\beta$-HCG) y ecografía obstétrica transvaginal realizada por un operador experimentado. El tratamiento

1 Médico, especialista en Ginecología y Obstetricia, Departamento de Ginecología y Obstetricia, Clínica Universidad de La Sabana, Chía (Colombia).

2 Médico General, Universidad Tecnológica de Pereira, residente de primer año de Ginecología y Obstetricia, Universidad de La Sabana, Departamento de Ginecología y Obstetricia, Clínica Universidad de La Sabana, Chía (Colombia).carlostrra@unisabana.edu.co generalmente es quirúrgico, con enfoque por vía laparoscópica o laparotomía exploratoria de acuerdo con la experiencia y el entrenamiento del cirujano tratante. No hay evidencia que respalde el uso de metrotexate en esta condición.

Conclusión: el embarazo ectópico ovárico es un cuadro clínico de presentación muy poco frecuente, el ultrasonido tiene utilidad limitada y el tratamiento quirúrgico con resección en cuña o la ooforectomía es el más utilizado.

Palabras clave: embarazo ectópico, embarazo ovárico, complicaciones del embarazo.

\section{ABSTRACT}

Objective: To report one case of ectopic ovarian pregnancy, and to review the literature on the diagnosis and treatment of this condition.

Materials and methods: An ectopic ovarian pregnancy in a patient using a copper intrauterine device (IUD) is reported. Laparoscopic treatment was provided in a private, level III general care institution. A search was conducted on the subject of the review in the PubMed, Ovid, ScienceDirect and Lilacs databases using the MeSH terms "pregnancy, ectopic"; "pregnancy, ovarian”; “pregnancy complications". The search included reports, case series and review articles.

Results: Overall, 23 references were found in the literature, including one book chapter, 9 topic reviews, and 13 reports or case series. 
Diagnosis requires a high degree of clinical suspicion, measurement of the beta subunit of the human chorionic gonadotropin hormone $(\beta-H C G)$, and transvaginal obstetric ultrasound performed by an experienced operator. Treatment is usually surgical through a laparoscopic approach or exploratory laparotomy, depending on the experience and training of the treating surgeon. There is no evidence supporting the use of methotrexate for this condition.

Conclusion: Ectopic ovarian pregnancies are infrequent clinical occurrences, ultrasound is of limited usefulness, and surgical treatment with wedge resection or oophorectomy is the most frequent form of management.

Key words: Ectopic pregnancy, ovarian pregnancy, complications of pregnancy.

\section{INTRODUCCIÓN}

El embarazo ectópico ovárico ocurre cuando el saco gestacional en desarrollo se implanta en uno de los dos ovarios. Esta condición representa 1,3$2 \%$ de todas las gestaciones reportadas (1), lo cual convierte al de localización ovárica en una variante rara, que reporta una incidencia de 1-3\% de todos los embarazos ectópicos diagnosticados $(2,3)$. La incidencia de este tipo de gestación ectópica ha venido en aumento en los últimos años, dado el advenimiento del uso de técnicas de reproducción asistida, las cuales constituyen el factor de riesgo más importante, con una incidencia de $0,3 \%$ por embarazo diagnosticado (4).

Para distinguir embarazos ectópicos ováricos de aquellos originados en las trompas uterinas se utilizan criterios histopatológicos estrictos. Los postulados por Spielberg en 1878 (5), describen: a) la trompa uterina del lado afectado debe estar indemne; b) el saco gestacional debe estar posicionado en el ovario; c) el ovario debe estar conectado al útero por el ligamento uteroovárico; d) debe demostrarse tejido ovárico en el saco gestacional extirpado.

Esta condición es un reto para el obstetra en el proceso diagnóstico del sangrado del primer trimestre del embarazo, ya que los signos y síntomas producidos por el mismo son muy similares al embarazo tubárico y al quiste de cuerpo lúteo roto (6). Por otra parte, el pronóstico posterior al tratamiento quirúrgico adecuado del embarazo ovárico difiere del pronóstico del embarazo tubárico en cuanto el primero no se asocia con un aumento del riesgo de ectópico en gestaciones subsiguientes, el cual sí se incrementa cuando se efectúa una salpingectomía para el manejo de un embarazo ectópico tubárico (4). A continuación, se presenta un raro caso de embarazo ectópico de implantación ovárica con el objetivo de revisar el enfoque diagnóstico y el tratamiento.

\section{PRESENTACIÓN DEL CASO}

Paciente de 32 años de edad, procedente de zona rural, que fue remitida a la Unidad de Ginecología y Obstetricia de la Clínica Universidad de La Sabana, institución privada de tercer nivel de complejidad del municipio de Chía, ubicado al norte de Bogotá, que atiende pacientes pertenecientes al aseguramiento contributivo en el sistema de salud de Colombia. Consulta por un cuadro clínico de 10 horas de evolución, con dolor abdominal hipogástrico tipo cólico, de intensidad moderada, asociado a dos episodios sincopales. Por la fecha de la última menstruación se calcula un retraso menstrual de 5 semanas más 5 días. Gestación 4 para 3, abortos 0 , antecedente familiar de hipertensión arterial, sin otros signos patológicos o psicosociales relevantes. Anota planificación familiar con DIU de cobre, desde su último parto 9 años atrás. Al examen físico se encuentra una paciente con facies de dolor intenso y estable hemodinámicamente. Como signos positivos se evidencia un abdomen blando, depresible, doloroso a la palpación profunda en hipogastrio y fosa iliaca derecha, sin signos de irritación peritoneal, con peristaltismo positivo. Posterior a ello se realiza especuloscopia que evidencia cuello de aspecto sano, hilos del DIU bien posicionados, con salida de flujo vaginal en escasa cantidad, de color amarillo y fétido. Al tacto vaginal bimanual se evidencia un 
útero en anteversoflexión, sin aumento de su tamaño, con dolor moderado a la palpación del anexo derecho. El resto del examen físico sin alteraciones aparentes. Se toman paraclínicos: fracción $\beta$ de gonadotropina coriónica humana (HCG) de 1157 $\mathrm{mUI} / \mathrm{ml}$ y ultrasonografía obstétrica transvaginal que revela una imagen anexial izquierda de $64 \mathrm{x}$ $36 \mathrm{~mm}$, con líquido laminar ecogénico atribuible a embarazo ectópico, DIU en posición intrauterina adecuada. Dados los anteriores hallazgos, la paciente es llevada a laparoscopia, con impresión diagnóstica de embarazo ectópico dependiente del anexo izquierdo frente a cuerpo lúteo hemorrágico, donde se documentan los siguientes hallazgos: hemoperitoneo de aproximadamente 300 cc. Se aprecia una masa en el polo superior del ovario derecho con sangrado activo de intensidad leve, de características macroscópicas similares al tejido trofoblástico (figura 1), la cual es resecada (figura 2) y rotulada para análisis histopatológico; anexo izquierdo indemne. Se da de alta y se realiza control de HCG en 48 horas, sin aumento y en franca disminución con respecto al valor inicial (165 mUI/ $\mathrm{ml})$. El espécimen fue caracterizado como tejido trofoblástico durante el análisis histopatológico, con tejido ovárico en su borde inferior. Evolución sin complicaciones.

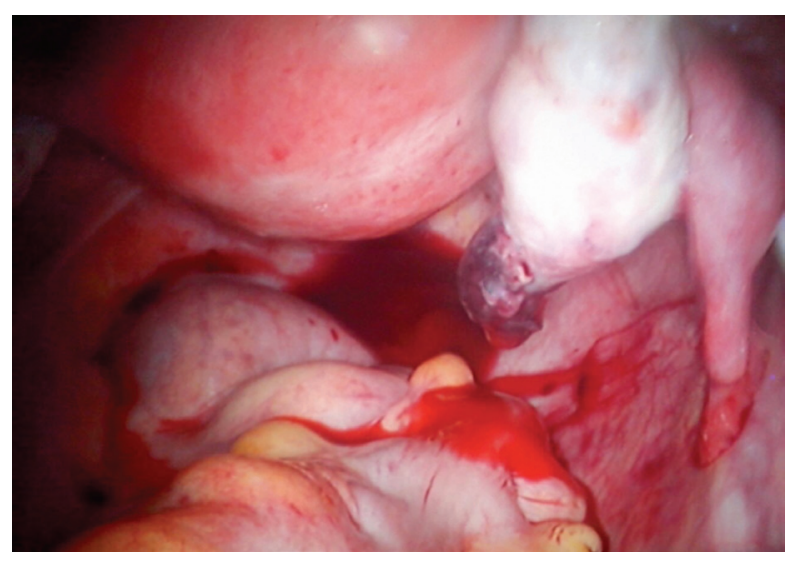

Figura 1. Masa ovárica con sangrado activo.

\section{MATERIALES Y MÉTODOS}

Con base en la siguiente pregunta: ¿qué hay de nuevo en el diagnóstico y tratamiento del embarazo ectópico ovárico?, se realizó búsqueda de la información médica en bases de datos PubMed, Ovid, ScienceDirect y Lilacs, mediante terminología MeSH: "pregnancy, ectopic"; "pregnancy, ovarian”; "pregnancy complications”. Se buscaron artículos de revisión narrativa, y reportes y series de caso en pacientes con embarazo ectópico ovárico. Se extrajo información sobre el tiempo de duración de los síntomas, el método para el diagnóstico, los hallazgos ultrasonográficos, quirúrgicos e histológicos, y el pronóstico de fertilidad posterior. La información se presenta de manera ordenada de acuerdo con los aspectos relevantes desde el punto de vista semiológico, diagnóstico y terapéutico.

Aspectos éticos. Se llevó a cabo el diligenciamiento del consentimiento informado dirigido. La paciente autorizó la publicación del caso clínico y se guardó la confidencialidad y privacidad de la información.

\section{RESULTADOS}

Se encontraron 23 referencias bibliográficas de las cuales una es un capítulo de libro, 9 son revisiones de tema y 13 son reportes o series de casos.

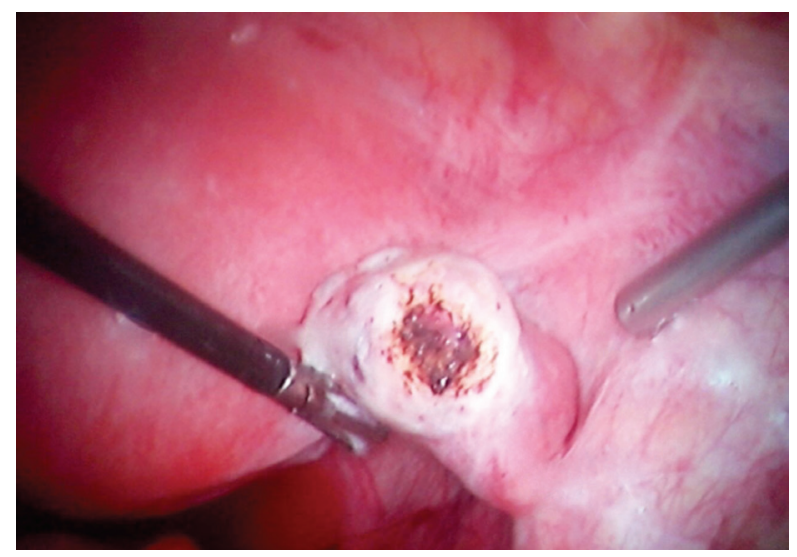

Figura 2. Superficie cauterizada posterior a resección de la masa. 
Diagnóstico. Un estudio que presenta 110 casos de embarazo ectópico ovárico informa que, en orden de frecuencia, los síntomas se presentan de la manera siguiente: dolor abdominal bajo intermitente (89,1\%), manchado (spotting) (47,3\%), masa anexial palpable $(38,2 \%)$, dolor lumbar $(28,6 \%)$ y choque $(11,8 \%)(4)$. A pesar de que la localización ovárica puede permitir un margen de crecimiento mayor, la ruptura en un estadio temprano es usualmente la consecuencia final en este tipo de gestación (7). Una proporción considerable de casos de embarazo ovárico son hallazgos intraoperatorios, cuando la paciente se presenta con una ruptura del ectópico y en estado de colapso circulatorio. Asimismo, en cirugía puede hacerse un dictamen inicial sugestivo de un cuerpo lúteo sangrante o un quiste del cuerpo lúteo hemorrágico (8). La ecografía juega un rol importante $(7,9)$. Sonográficamente resalta la presencia de un anillo ecogénico amplio sobre el ovario, en comparación con el tejido ovárico (10). El hallazgo de una vesícula vitelina o partes fetales son los marcadores ultrasonográficos claves para el diagnóstico; sin embargo, la visualización de un embrión dentro del quiste es infrecuente (11). Los criterios establecidos por Spiegelberg no son extrapolables a la ecografía, por lo cual el reto es distinguir por esta vía si se trata de un embarazo ovárico, un cuerpo lúteo o un quiste hemorrágico (12). Otros hallazgos ecográficos son el aumento de tamaño del ovario y líquido alrededor del mismo (13). El uso del Doppler color no parece aportar al diagnóstico según Atri (14). El encontró que un índice de resistencia $<0,39$ tiene una especificidad del $100 \%$, pero con una sensibilidad de solo un 15 \% para embarazo ectópico, por lo cual concluye que índices de resistencia bajos y altos ayudan a discriminar un embarazo ectópico de un quiste de cuerpo lúteo, pero estos hallazgos solo se presentan en una de cada 10 pacientes (14). Se ha reportado que la resonancia magnética nuclear podría ser útil en el diagnóstico del embarazo ovárico, ya que permite identificar la exacta localización de la masa, cambios por la hemorragia en el ovario y la ausencia de alteraciones en la trompa uterina (15).

La cirugía (laparoscopia o laparotomía) permanece como el estándar de diagnóstico y manejo (6). Al momento de la cirugía el diagnóstico puede ser realizado mediante análisis histopatológico de la muestra si no se aprecian partes fetales, por ejemplo, en embarazos muy pequeños (16).

Tratamiento. En cuanto al manejo de la entidad, los enfoques actuales están basados en experiencias presentadas en reportes de caso y análisis retrospectivos. En general, la cirugía es la perspectiva de tratamiento más utilizada, siendo la laparoscopia el estándar de oro de tratamiento.

En el estudio antes mencionado de Ko et al. (4), se encontró que un 70,9\% de las pacientes fueron intervenidas por vía laparoscópica contra un 29,1\% por laparotomía exploratoria. El $93 \%$ requirió resección en cuña de la lesión y el 7 \% ooforectomía. Al comparar los dos enfoques se halló que la cirugía mínimamente invasiva se asocia a menor tiempo quirúrgico, menor pérdida sanguínea y menor tiempo de estancia hospitalaria comparada con el enfoque por laparotomía. Las técnicas quirúrgicas para la resección en cuña varían. Una técnica descrita por Nadarajah et al., involucra asegurar el ovario y realizar una disección aguda y roma del ectópico, haciendo hemostasia con electrocauterio (17). Otra técnica que utiliza cauterio monopolar o bipolar ha sido descrita por Gavrilova-Jordan et al.; en esta, se realiza una disección por producción de calor local con hemostasia por medio de diatermia. Se usan lavados con solución salina para disminuir la temperatura del tejido (18). Eskandar et al., han utilizado ondas de ultrasonido de alta frecuencia para coagular y cortar el tejido de manera simultánea con menor tasa de daño colateral comparado con el electrobisturí, asociándose a mayor preservación de tejido ovárico (19). Infortunadamente, son dispositivos muy costosos y no disponibles en todos los países.

Otra opción de manejo es la terapia médica con metotrexate. Un reporte de caso describe el 
manejo con dosis habituales $(1 \mathrm{mg} / \mathrm{kg}$ de peso por vía intramuscular, dosis única), que requirió nueva dosis al séptimo día por falta de descenso de HCG con posterior evolución satisfactoria (20). Otro reporte reciente relata por primera vez el manejo con el metotrexate inyectado localmente, guiado por ecografía, con resultados satisfactorios a los 7 días; sin embargo, no hay estudios que avalen su uso y pronóstico en esta condición (21). Además, es probable que las inyecciones de fármacos provoquen sangrado masivo del ovario (4). Hasta el momento, es incierta la tasa de éxito con el manejo médico.

Pronóstico. No ha sido reportado ningún caso de embarazo ectópico ovárico recurrente con el uso de la cirugía (22). Un estudio llevado a cabo por Portuondo et al., encontró que las pacientes tratadas mediante cirugía por esta condición presentan una alta tasa reproductiva ulterior, logrando el embarazo intrauterino viable a los 18 meses del posoperatorio en quienes no utilizan algún método anticonceptivo (23).

\section{CONCLUSIONES}

El embarazo ectópico ovárico es una condición rara que amerita ser tenida en cuenta durante el enfoque diagnóstico de una paciente con sangrado del primer trimestre. La utilidad del ultrasonido es limitada. Usualmente, requiere manejo quirúrgico mediante resección en cuña u ooforectomía, siendo el enfoque por vía laparoscópica el más utilizado en los últimos tiempos.

\section{AGRADECIMIENTOS}

$\mathrm{Al}$ personal del área de archivo y del área de patología de la Clínica Universidad de La Sabana, quienes facilitaron la historia clínica y el resultado del análisis histopatológico en medio magnético para su posterior utilización en la estructuración del presente reporte.

\section{REFERENCIAS}

1. Saraiya M, Berg CJ, Shulman H, Green CA, Atrash HK. Estimates of the annual number of clinically recognized pregnancies in the United States, 1981-1991. Am J Epidemiol. 1999;149:1025-9.

2. Einenkel J, Baier D, Horn LC, Alexander H. Laparoscopic therapy of an intact primary ovarian pregnancy with ovarian hyperstimulation syndrome: case report. Hum Reprod. 2000;15:2037-40.

3. Raziel A, Schachter M, Mordechai E, Friedler S, Panski M, Ron-El R. Ovarian pregnancy-a 12-year experience of 19 cases in one institution. Eur J Obstet Gynecol Reprod Biol. 2004;114:92-6.

4. Ko PC, Lo LM, Hsieh TT, Cheng PJ. Twenty-one years of experience with ovarian ectopic pregnancy at one institution in Taiwan. Int J Gynaecol Obstet. 2012;119:154-8.

5. Spiegelberg O. Casuistik der ovarialcshwangerschaft. Arch Gynaekol. 1878; 13:73-7.

6. Ghasemi Tehrani H, Hamoush Z, Ghasemi M, Hashemi L. Ovarian ectopic pregnancy: A rare case. Iran J Reprod Med. 2014;12:281-4.

7. Cunningham F, Leveno KJ, Bloom SL, Spong CY, Dashe JS, Hoffman BL, et al. Ectopic Pregnancy. En: Cunningham F, Leveno KJ, Bloom SL, Spong CY, Dashe JS, Hoffman BL, et al. eds. Williams Obstetrics. Twenty-Fourth Edition. New York: McGraw-Hill; 2013.

8. Goyal LD, Tondon R, Goel P, Sehgal A. Ovarian ectopic pregnancy: A 10 years' experience and review of literature. Iran J Reprod Med. 2014;12:825-30.

9. Choi HJ, Im KS, Jung HJ, et al. Clinical analysis of ovarian pregnancy: a report of 49 cases. Eur J Obstet Gynecol Reprod Biol. 2011;158:87.

10. Comstock C, Huston K, Lee W. The ultrasonographic appearance of ovarian ectopic pregnancies. Obstet Gynecol. 2005;105:42-5.

11. Dimitrov R, Karag'ozov I, Filipov E, Radulov N. A case of unruptured intrafollicular ovarian pregnancy diagnosed prior to operation. Akush Ginekol (Sofiia). 2002;41:44-7.

12. Stein MW, Ricci ZJ, Novak L, Robert SJH, Koenigsberg M. Sonographic comparison of the tubal ring of ectopic pregnancy with the corpus luteum. J Ultrasound Med. 2004;23:57-62.

13. Choi HJ, Im KS, Jung HJ, Lim KT, Mok JE, Kwon YS. Clinical analysis of ovarian pregnancy: a report of 49 cases. Eur J Obstet Gynecol Reprod Biol. 2011;158:87-9. 
14. Atri M. Ectopic pregnancy versus corpus luteum cyst revisited: best Doppler predictors. J Ultrasound Med. 2003;22:1181-4.

15. Io S, Hasegawa M, Koyama T. A case of ovarian pregnancy Diagnosed by MRI. Case Rep Obstet Gynecol. 2015;2015:143031.

16. Kraemer B, Kraemer E, Guengoer E, Juhasz-Boess I, Solomayer EF, Wallwiener D, Rajab TK. Ovarian ectopic pregnancy: diagnosis, treatment, correlation to Carnegie stage 16 and review based on a clinical case. Fertil Steril. 2009;92:392.e13-5.

17. Nadarajah S, Sim LN, Loh SF. Laparoscopic management of an ovarian pregnancy. Singapore Med J. 2002;43:095-6.

18. Gavrilova-Jordan L, Tatpati L, Famuyide A. Primary ovarian pregnancy after donor embryo transfer: early diagnosis and laparoscopic treatment. JSLS. 2006;10:70-3.
19. Eskandar O. Conservative laparoscopic management of a case of ruptured ovarian ectopic pregnancy by using a Harmonic scalpel. J Obstet Gynaecol. 2010;30:67-9.

20. Kiran G, Guven AM, Köstü B. Systemic medical management of ovarian pregnancy. Int J Gynaecol Obstet. 2005;91:177-8.

21. Pagidas K, Frishman GN. Nonsurgical management of primary ovarian pregnancy with transvaginal ultrasound-guided local administration of methotrexate. J Minim Invasive Gynecol. 2013;20:252

22. Joseph RJ, Irvine LM. Ovarian ectopic pregnancy: aetiology, diagnosis, and challenges in surgical management. J Obstet Gynaecol. 2012;32:472-4.

23. Portuondo JA, Ochoa C, Gomez BJ, Uribarren A. Fertility and contraception of 6 patients with ovarian pregnancy. Int J Fertil. 1984; 29:254-6. 